\title{
NFL and CXCL13 may reveal disease activity in clinically and radiologically stable MS
}

Novakova $\mathrm{L}^{1}$, Axelsson $\mathrm{M}^{1}$, Malmeström $\mathrm{C}^{1}$, Zetterberg $\mathrm{H}^{2,4,5,6}$, Blennow $\mathrm{K}^{2,4}$, Svenningsson $\mathrm{A}^{3}$, Lycke $\mathrm{J}^{1}$

${ }^{1}$ Department of Clinical Neuroscience, Institute of Neuroscience and Physiology, Sahlgrenska Academy, Gothenburg

${ }^{2}$ Department of Psychiatry and Neurochemistry, Institute of Neuroscience and Physiology, Sahlgrenska Academy, Gothenburg

${ }^{3}$ Department of Clinical Sciences Danderyd Hospital, Karolinska Institutet, Stockholm

${ }^{4}$ Clinical Neurochemistry Laboratory, Sahlgrenska University Hospital, Mölndal, Sweden

${ }^{5}$ Department of Neurodegenerative Disease, UCL Institute of Neurology, Queen Square, London, UK

${ }^{6}$ UK Dementia Research Institute at UCL, London, UK

\section{Keywords}

Multiple sclerosis, biomarker, cerebrospinal fluid, disease activity, neurofilament light, CXCL13

\section{Corresponding author}

Lenka Novakova, MD, $\mathrm{PhD}$

E-mail: lenka.novakova@vgregion.se, lenka.novakova@neuro.gu.se

Telephone number: +46730518041

Word count: abstract 299, manuscript (main text) 2647 (excluding Tables, Figure legends, Acknowledgement and References)

Running title: NFL and CXCL13 may reveal disease activity in clinically and radiologically stable MS

\section{Disclosure of Conflict of Interest statement}

AS reports no conflicts of interests.

CM has received honoraria for lectures and advisory boards from Biogen, Merck Novartis, Roche and Sanofi. HZ has served at scientific advisory boards for Denali, Roche Diagnostics, Wave, Samumed and CogRx, has given lectures in symposia sponsored by Fujirebio, Alzecure and Biogen, and is a co-founder of Brain Biomarker Solutions in Gothenburg AB (BBS), which is a part of the GU Ventures Incubator Program.

JL has received travel support and/or lecture honoraria from Biogen, Novartis, Merck, Roche and SanofiGenzyme; has served on scientific advisory boards for Biogen, Novartis, Merck, Alexion, Roche and SanofiGenzyme; serves on the editorial board of the Acta Neurologica Scandinavica; has received unconditional research grants from Biogen and Novartis.

$\mathrm{KB}$ has served as a consultant or at advisory boards for Alzheon, Eli-Lilly, Fujirebio Europe, IBL International and Roche Diagnostics, and is a co-founder of Brain Biomarker Solutions in Gothenburg AB, a GU Venturesbased platform company at the University of Gothenburg.

LN has received honoraria for lecture from Biogen and Novartis, for advisory boards from Merck.

MA has received honoraria for lectures and/or advisory boards from Biogen, Genzyme, and Novartis. 


\begin{abstract}
Background: Cerebrospinal fluid (CSF) levels of neurofilament light (NFL), a biomarker of axonal damage, and CXCL13, a chemokine involved in B-cell regulation, are both associated with disease activity in multiple sclerosis (MS).

Objective: To explore the potential of NFL and CXCL13 to detect residual disease activity in patients with no signs of clinical or ongoing radiological activity and to study the clinical relevance of such activity.

Methods: NFL and CXCL13 concentrations were determined with ELISA in CSF obtained from 90 relapsingremitting (RR) MS and 47 Progressive (Pr) MS (including primary and secondary PrMS) at baseline and after 12 months of follow-up. The patients were assessed at baseline, before initiating or switching disease modifying therapy (DMT) and again after 12 and 27 months of follow-up.
\end{abstract}

Results: All patients with ongoing disease activity (relapse or contrast-enhancing lesions on MRI) had increased NFL or CXCL13. The proportion of RRMS and PrMS patients without ongoing disease activity with elevation of either NFL or CXCL13 (residual disease activity) was 39\% and 50\%, respectively, and both were increased in $11 \%$ and $16 \%$, respectively. The treatment with DMTs decreased the proportion with residual disease activity in both RRMS and PrMS significantly. We could not show any significant association between residual disease activity and clinical or MRI measures at 12 or 27 months of follow-up.

Conclusions: Although most of this real-world study population had been treated with second-line DMTs and achieved clinical and radiological stability, a significant proportion of patients still displayed increased CSF levels of both NFL and CXCL13, indicating residual disease activity. Thus, these markers seemed considerably more sensitive to disease activity than clinical and MRI measures. However, the long-term clinical significance of such activity remains to be determined.

2 NFL and CXCL13 reveal disease activity in clinically and radiologically stable MS 


\section{Introduction}

The current assessment of disease activity, disability and therapeutic response in multiple sclerosis (MS) relies essentially on clinical and magnetic resonance imaging (MRI) measures. However, the low relapse rate during disease-modifying therapies (DMTs) and the limited reliability of disability assessment (Meyer-Moock et al. 2014) make clinical measures insensitive. Although, conventional monitoring of MS with cerebral MRI may detect new or enlarging lesions, as signs of inflammatory activity, clinically silent myelitis, diffuse inflammation and demyelination and neuro-axonal degeneration, may escape detection (Lycke \& Zetterberg 2017). Thus, there is an unmet need to improve the assessment of patients with MS, particularly for evaluating DMT response. We and others have shown that biomarkers of inflammation and degeneration in cerebrospinal fluid (CSF) may add information that is clinically useful (Dubuisson et al. 2017).

Levels of neurofilament light protein (NFL) in the CSF, a marker of axonal damage, can be used to monitor neurodegeneration (Teunissen \& Khalil 2012). However, elevated levels of NFL are primarily associated with disease activity in MS (Malmestrom et al. 2003), supporting that axonal damage in MS is secondary to the immune attack of the CNS that characterizes the disease. The chemokine CXCL13, also known as B cell-attracting chemokine 1 (BCA-1), is a regulator of B-cell activity. The levels of NFL and CXCL13 in CSF are increased during relapse and are reduced after treatment with DMTs (Sellebjerg et al. 2009, Gunnarsson et al. 2011). In MS, CSF-CXCL13 levels correlate with CSF-NFL levels (Novakova et al. 2017a), again supporting an association between B cell driven inflammation and axonal injury in the pathogenesis of MS.

Here, we investigate the degree of inflammation and axonal injury in relapsing-remitting multiple sclerosis (RRMS) and progressive multiple sclerosis (PrMS) patients, classified as active or inactive using standard clinical or imaging criteria, by comparing CSF levels of NFL and CXCL13 in the different groups. The purpose was to determine if these biomarkers could improve the evaluation of patients, treated with DMTs, by revealing signs of residual disease activity in patients who are considered clinically and radiologically stable.

\section{Materials and Methods}

Patients and clinical assessment and of disease activity

Patients with MS were enrolled in the study at the neurology departments of University Hospital of Umeå, Umeå and Sahlgrenska University Hospital, Gothenburg, Sweden. All patients participated voluntarily in the study and 3 NFL and CXCL13 reveal disease activity in clinically and radiologically stable MS 
informed consent was obtained from all subjects after oral and written information was provided. The Regional Ethical Review Board of Uppsala approved the study. The patients in this study had previously participated in studies on biomarkers (Novakova et al. 2017b, Gunnarsson et al. 2011).

The study population consisted of 90 patients with RRMS and 47 with PrMS. The latter group included 7 with primary progressive (PP) MS and 40 with secondary progressive (SP) MS. Patients were included in the study at treatment initiation or treatment switch and if they consented to perform lumbar puncture twice. Patients were followed prospectively and were re-examined twice, the first time after a median of 12 months (IQR 10-14 months), and the second time after a median of 27 months (IQR 24-35 months) from baseline. At baseline, 81 patients were treated and 56 were untreated, including 39 treatment-naïve patients and 17 patients previously on DMT with washout periods more than 3 months. These patients initiated their first DMT $(n=46)$, escalated treatment $(n=62)$ or changed to DMTs with the same efficacy $(n=19)$. At 12 months of follow up, 10 patients were untreated and 127 (88 RRMS and 39 PrMS) were on DMTs: natalizumab ( $n=55)$, rituximab ( $\mathrm{n}=23)$, fingolimod $(n=21)$, mitoxantrone $(n=13)$, methotrexate $(n=7)$, interferon beta $(n=2)$, alemtuzumab $(n=2)$, glatiramer acetate

$(n=2)$, glatiramer acetate+mitoxantrone $(n=1)$ and cyclophosphamide $(n=1)$.

Patients were assessed by clinical neurological examination and disability was scored by the Expanded Disability Status Scale (EDSS) (Kurtzke 1983). Disability was improved if EDSS decreased $\geq 1.0$-point when baseline EDSS was 1.0-5.5 or $\geq 0.5$ when baseline EDSS was $\geq 6$, and was worsened if EDSS increased $\geq 1.0$-point if baseline EDSS was $0.0-5.0$ or $\geq 0.5$ if baseline EDSS was $\geq 5.5$, and was considered unchanged if EDSS changed less. Ongoing disease activity was defined as a relapse within 3 months prior to sampling and/or one or more gadolinium (Gd) contrast enhancing lesions on MRI performed 6 weeks before or after sampling (Cotton et al. 2003). No evidence of disease activity (NEDA) was defined as no relapse, no increase in disability measured by EDSS, no new or enlarging T2 lesions and no Gd contrast enhancing lesions on MRI. Residual disease activity was defined as increased NFL and/or CXCL13 in CSF in patients with no signs of clinical (relapse) or MRI activity (contrast or new or enlarging non-contrast enhancing lesion). The study design is presented in Figure 1 . The summary of patients' demographic and clinical characteristics is presented in Table 1.

\section{CSF sampling and immunoassays of NFL and CXCL13}

CSF samples were obtained at baseline and at the first re-examination of patients with median 12 months followup, which resulted in a total of 274 samples. Lumbar puncture and CSF was handled according to the consensus protocol of the BioMS-EU network for CSF biomarker research in MS (Teunissen et al. 2009). Licensed laboratory 
technicians in the Clinical Neurochemistry Laboratory at Sahlgrenska University Hospital performed the biomarker analyses.

CSF NFL concentration was measured with a sandwich ELISA (NF-light@ ELISA kit, UmanDiagnostics AB, Umeå, Sweden). Intra- and inter-assay coefficients of variation were below $10 \%$. The lower limit of quantification (LLoQ) of the assay was $31 \mathrm{ng} / \mathrm{L}$. NFL is age-dependent and considered increased if concentrations were $<30$ years: $<380 \mathrm{ng} / \mathrm{L} ; 30$ years- $<40$ years: $<560 \mathrm{ng} / \mathrm{L} ; 40$ years- $<60$ years: $<890 \mathrm{ng} / \mathrm{L} ; \geq 60$ years: $<1850 \mathrm{ng} / \mathrm{L}$ (in house normal reference values).

CSF CXCL13 was determined with ELISA (Human CXCL13/BLC/BCA-1 Immunoassay, R\&D Systems Inc., Abingdon, United Kingdom), according to the manufacturer's instructions. The average intra-and inter-assay coefficients of variation were below $10 \%$ and the LLoQ was $0.2 \mathrm{pg} / \mathrm{mL}$. CXCL13 concentrations were considered increased above the reference value of $7.8 \mathrm{ng} / \mathrm{L}$ (ref: https://www.ncbi.nlm.nih.gov/pmc/articles/PMC3546953/).

\section{Statistics}

Statistical analysis was performed using Microsoft Office Excel 2016 and IBM SPSS Statistics software 26. The Wilcoxon signed rank sum test was used for analysis of matched pair data, i.e., before and after treatment. Binary logistic regression was used to predict the odds of disease activity at follow-up based on biomarkers. Correlations were determined with Spearman's rank correlation coefficient.

\section{Results}

As expected, patients with PrMS were older, had longer disease duration, higher disability and included a lower female/male ratio than those with RRMS course (Table 1). The age-dependent biomarker NFL was higher in PrMS than in RRMS while CXCL13 showed the opposite pattern (Table 4). However, the proportion of patients with increased levels of these biomarkers above reference values of normality was similar in RRMS and PrMS (Table 2). The proportion of active versus inactive RRMS and PrMS was almost equally distributed, but more RRMS $(80 \%)$ than PrMS $(55.3 \%)$ were previously on DMT (Table 1).

\section{Biomarker relation to disease activity}

All samples from patients with ongoing disease activity had increased levels of NFL or CXCL13. In RRMS and PrMS without ongoing disease activity, the increase of either NFL or CXCL13 were found in 39.3\% and 50\%, and both were increased in $10.9 \%$ and $16.4 \%$ of patients, respectively (Table 2). Thus, no major differences were found 5 NFL and CXCL13 reveal disease activity in clinically and radiologically stable MS 
between RRMS and PrMS. We analyzed the contribution of each biomarker to detect residual disease activity (Table 3). In patients without ongoing disease activity and with normal NFL, 5.7\% of RRMS and 9.8\% of PrMS had increased CXCL13 and in patients with normal CXCL13, 21\% RRMS and 19.3\% PrMS had increased NFL, indicating that NFL was more sensitive. The levels of NFL and CXCL13 in CSF correlated with Spearman's rho $0.415(\mathrm{p}<0.001)$

The predictive value of residual activity in patients without ongoing disease activity on outcome

We analyzed if residual disease activity, i.e. increased biomarker levels at baseline in patients without ongoing disease activity influenced clinical or MRI outcome at follow-up 12 or 27 months later. The increase of NFL or CXCL13 at baseline seemed not predict relapse, MRI lesion formation, worsening in EDSS, nor NEDA at followup, nor could we show their predictive value using 12 month assessment as re-baseline and follow-up 15 months later (Table 5).

\section{Biomarker response to treatment intervention}

The treatment with various DMTs reduced the levels of CSF NFL in $87.2 \%$ of RRMS and in $79.5 \%$ of PrMS patients; and the levels of CSF CXCL13 in $95.5 \%$ of RRMS and in $89.7 \%$ of PrMS patients (Figure 2). Biomarker levels were reduced at follow-up irrespective of ongoing therapy, disease activity, or disability development (Table 4). Again, the reduction of biomarker levels were similar in RRMS as compared with PrMS. Although rituximab is a B-cell depleting treatment, the reduction of CSF CXCL13 was not different in patients treated with rituximab compared to those on other DMTs ( $\mathrm{p}=0.028$ and $\mathrm{p}<0.001$, respectively). The proportion of previously untreated patients with ongoing disease activity (23 RRMS and 25 PrMS) decreased from $56.3 \%$ at baseline to $16.7 \%$ at 12 months of follow up. In previously treated patients (65 RRMS and 14 PrMS), the proportion of patients with ongoing disease activity decreased from $40.5 \%$ at baseline to $8.9 \%$ at 12 months of follow up. After treatment, the proportion of patients with residual activity i.e. increased NFL or CXCL13 in patients without relapse or Gd enhancement on MRI was $21.3 \%$ or $3.9 \%$ in RRMS and $22.2 \%$ or $13.9 \%$ in PrMS, respectively.

\section{Discussion}

The aim was to study the utility of NFL and CXCL13 as biomarkers in MS to reveal residual disease activity in patients lacking clinical activity or contrast enhancement on conventional cerebral MRI. We confirmed previous association between levels of NFL and CXCL13 in CSF and disease activity (Malmestrom et al. 2003, Khademi 6 NFL and CXCL13 reveal disease activity in clinically and radiologically stable MS 
et al. 2011) and response to DMT intervention (Sellebjerg et al. 2009, Gunnarsson et al. 2011). However, the novel finding in this study is the increased levels of these biomarkers in patients who are considered to be stable and do not show any signs of ongoing disease activity. In contrast, previous studies show that NFL and CXCL13 are increased in only $5 \%$ and $0 \%$ of healthy controls, respectively (Novakova et al. 2017a). Interestingly, we found no differences in the biomarker levels, their association to disease activity, or their response to DMTs between patients of relapsing and progressive course. Our results were obtained from a real world clinical setting and thus indicate that NFL and CXCL13 might contribute in the assessment of patients and for monitoring the therapeutic response in MS.

There is convincing evidence that support an effect of DMTs on both inflammatory activity and degenerative processes in MS (Ziemssen et al. 2015). By analyzing different biomarkers in CSF that reflect different parts of the pathogenesis in MS, it should be possible to determine which processes that are affected during therapeutic intervention. In this study we combined NFL, a marker of axonal damage and degeneration with CXCL13, a chemokine that is involved in B-cell regulation. Our results show that there might be residual axonal degeneration in patients without clinical or MRI activity. However, both NFL and CXCL13 seemed to be markers of disease activity and were reduced during therapeutic intervention. No major differences were found between RRMS and PrMS suggesting that axonal degeneration is mostly secondary to inflammation also in PrMS.

The present study provides further evidence that the effects of DMTs in MS can be monitored using CSF biomarkers. The modes of action of DMTs are diverse and may also be revealed by their influence on different biomarkers in CSF. CXCL13 is a chemokine that is involved in B-cell regulation (Kowarik et al. 2012). It binds to the receptor CXCR5 and plays essential role in inflammatory, infectious and immune responses (Kazanietz et al. 2019). CXCL13 seem also to contribute in the formation of ectopic lymphoid tissues found in MS, in particular in SPMS (Mitsdoerffer \& Peters 2016). We showed a significant reduction of CXCL13 by treatment with rituximab as well as with other DMTs. Thus, our results suggest that CXCL13 may have broader influence on MS inflammation than B-cell regulation solely.

Only few patients without ongoing disease activity and with normal CSF NFL levels had increased levels of CSF CXCL13. In contrast, around one fifth of patients without ongoing disease activity and with normal CXCL13 still showed signs of axonal degeneration. Both biomarkers were correlated to each other and hence, the contribution from CXCL13 seemed low. Further, although CXCL13 can be determined in both CSF and blood the CXCL13 concentration in these compartments may not reflect similar pathological processes (Pilz et al. 2020). Infections 
or other co-morbidities outside the CNS may influence blood CXCL13 (Kazanietz et al. 2019). In contrast, NFL is a biomarker specific for the nervous system (Norgren et al. 2003, Trapp et al. 1998) and due to the recent development of ultrasensitive immune-assays; NFL can be determined in serum and plasma ((Kuhle et al. 2016). The correlation between CSF and serum/plasma NFL is high (Disanto et al. 2017, Novakova et al. 2017b) and several investigations show that serum/plasma NFL respond from disease activity and therapeutic intervention as previously shown in CSF (Novakova et al. 2017b, Kuhle et al. 2019, Thebault et al. 2019, Sejbaek et al. 2019). Moreover, the temporal change of NFL concentrations after CNS injury seems similar in CSF and blood (Bergman et al. 2016). Although CSF NFL is more sensitive than serum/plasma NFL (Disanto et al. 2017), our results are likely to be similar in blood.

We found residual activity in patients without disease activity determined with conventional clinical and MRI methods. Although a majority of our prospectively followed patients achieved NEDA they still had increased levels of NFL or CXCL13. However, we could not show that such residual activity had a predictive value. Clinical or MRI outcome measures were not worse in patients with residual activity as compared with those without at a limited follow-up of 12 and 27 months from baseline. Thus, we cannot show evidence that support inclusion of these biomarkers in NEDA to improve prediction of outcome (Giovannoni et al. 2015). Previous studies have shown that NFL at onset may predict increased risk of brain and spinal cord atrophy at 2 and 5 years of follow-up (Barro et al. 2018, Hakansson et al. 2018). The potential impact on degeneration and disability from subclinical axonal degeneration probably deserves long-term studies.

One limitation of this study was the absence of MRI data on spinal lesions. Although, the majority of spinal lesions are considered to give rise to relapses, recent studies show that almost ??\% of active spinal lesions are clinically silent (REF). Another possible source of error we have not controlled for was the occurrence of cerebrovascular white matter disease that increases with age and may lead to increased levels of NFL (Bjerke et al. 2014), but not of CXCL13. However, since neither of these biomarkers differed between inactive RRMS and PrMS patients, the latter being significantly older, this is unlikely to be an important confounder in our study. This study is based on biomarkers in CSF with the disadvantage that only two samples per patient were available; measuring blood NFL repeatedly using new ultrasensitive Single molecule array technology (ref: PMID: 30171200) could shed additional light on whether or not axonal degeneration remains despite the therapy and when, in relation to therapy start, normalization starts to occur.

8 NFL and CXCL13 reveal disease activity in clinically and radiologically stable MS 
In summary, our data imply that there may be increased NFL and CXCL13 levels indicating axonal degeneration and inflammatory activity in CSF in patients without ongoing clinical or MRI activity. This finding supports the use of these biomarkers, as a complement to MRI and clinical examination to detect residual disease activity. We show that such activity was also present after DMTs. Interestingly, no difference was found in NFL and CXCL13 levels between RRMS and PrMS. However, these biomarkers could not predict forthcoming disease activity or disability development in our study with limited follow-up. Their clinical relevance remains to be explored in long-term investigations.

9 NFL and CXCL13 reveal disease activity in clinically and radiologically stable MS 
Table 1. Demographic and clinical characteristics of patients

\begin{tabular}{|c|c|c|c|c|c|c|}
\hline & $\begin{array}{c}\text { RRMS } \\
n=90\end{array}$ & & & $\begin{array}{l}\text { PrMS } \\
n=47\end{array}$ & & \\
\hline Age [years], mean(range) & $36(15-60)$ & & & $47(22-67)$ & & \\
\hline Gender (F/M) & $59 / 31$ & & & $24 / 23$ & & \\
\hline Disease duration, mean(range) & $8(0-26)$ & & & $15(0-38)$ & & \\
\hline \multirow[t]{2}{*}{ No treatment, $\mathrm{n}(\%)$} & $18(20)$ & & & $21(44.7)$ & & \\
\hline & Baseline & Follow-up 1 & Follow-up 2 & Baseline & Follow-up 1 & Follow-up 2 \\
\hline Ongoing disease activity $(\%)$ & 51.5 & 13.3 & & 41.9 & 10.6 & \\
\hline $\operatorname{NEDA}(\%)$ & & 70 & 71.8 & & 59.6 & 51.2 \\
\hline Relapse (\%) & 33.3 & 11.1 & 7.1 & 14.9 & 6.4 & 4.8 \\
\hline One or more $\mathrm{Gd}+$ lesion $(\%)$ & 31.5 & 6.7 & 1.2 & 34.9 & 9.1 & 21.2 \\
\hline EDSS, mean (range) & $2.6(0-6.5)$ & $2.3(0-6.5)$ & $2.4(0-6.5)$ & $5.6(2.5-8.5)$ & $6.0(2.5-9.0)$ & $5.9(2.5-9.0)$ \\
\hline
\end{tabular}

EDSS=Expanded Disability Status Scale, F=female, Gd+=gadolinium enhancing, $\mathrm{M=male}$, PrMS=progressive multiple sclerosis, RRMS=relapsing-remitting multiple sclerosis, activity=relapse and/or MRI with Gd+at lumbar puncture

Table 2. The proportion of patients with increased levels of NFL and/or CXCL13 including all samples

\begin{tabular}{ll|c|c|c|c} 
& & $\uparrow$ NFL & $\uparrow$ CXCL 13 $\uparrow$ NFL or $\uparrow$ CXCL 13 & $\uparrow$ NFL and $\uparrow$ CXCL 13 \\
\hline RRMS & no activity (n=122) & $27.5 \%$ & $12.3 \%$ & $39.3 \%$ & $10.9 \%$ \\
& with activity (n=58) & $60.7 \%$ & $51.7 \%$ & $100 \%$ & $58.8 \%$ \\
\hline PrMS & no activity (n=72) & $29.2 \%$ & $20.8 \%$ & $50 \%$ & $16.4 \%$ \\
& with activity (n=22) & $72.7 \%$ & $59.1 \%$ & $100 \%$ & $73.3 \%$ \\
\hline
\end{tabular}

CXCL13=C-X-C motif chemokine 13, NFL=neurofilament light, RRMS=relapsing-remitting multiple sclerosis, PrMS=progressive multiple sclerosis, activity= relapse and/or MRI with $\mathrm{Gd}+$ at lumbar puncture

Table 3. The ability of NFL and CXCL13 to detect residual activity in patients with and without clinical or MRI disease activity

\begin{tabular}{ll|c|l|c}
\multicolumn{2}{c|}{ normal NFL } & normal NFL and $\uparrow$ CXCL 13 & normal CXCL 13 & normal CXCL 13 and $\uparrow$ NFL \\
\hline RRMS & no activity $(\mathrm{n}=87)$ & $5.7 \%(\mathrm{n}=5)$ & no activity $(\mathrm{n}=107)$ & $21.9 \%(\mathrm{n}=24)$ \\
& with activity $(\mathrm{n}=22)$ & $36.4 \%(\mathrm{n}=8)$ & with activity $(\mathrm{n}=28)$ & $50 \%(\mathrm{n}=14)$ \\
\hline PrMS & no activity $(\mathrm{n}=51)$ & $9.8 \%(\mathrm{n}=5)$ & no activity $(\mathrm{n}=57)$ & $19.3 \%(\mathrm{n}=11)$ \\
& with activity $(\mathrm{n}=6)$ & $33.3 \%(\mathrm{n}=2)$ & with activity $(\mathrm{n}=9)$ & $55.6 \%(\mathrm{n}=5)$ \\
\hline
\end{tabular}

CXCL13=C-X-C motif chemokine 13, NFL=neurofilament light, RRMS=relapsing-remitting multiple sclerosis,

PrMS=progressive multiple sclerosis, activity= relapse and/or MRI with Gd+ at lumbar puncture. Residual activity is defined as a value above normal reference range.

10 NFL and CXCL13 reveal disease activity in clinically and radiologically stable MS 
Table 4. Biomarker response to treatment intervention

\begin{tabular}{|c|c|c|c|c|c|c|}
\hline & Baseline & Follow-up & $\mathrm{p}$ value & Baseline & Follow-up & $\mathrm{p}$ value \\
\hline & \multicolumn{3}{|l|}{$\begin{array}{l}\text { All RRMS } \\
(\mathrm{n}=90)\end{array}$} & \multicolumn{3}{|l|}{$\begin{array}{l}\text { All PrMS } \\
(n=47)\end{array}$} \\
\hline NFL[ng/L] median (IQR) & 665 (408-1078) & $371(265-528)$ & $<0.001$ & $805(560-1160)$ & $632(460-890)$ & 0.009 \\
\hline \multirow[t]{2}{*}{ CXCL13[ng/L] median (IQR) } & $5.4(0.2-19.4)$ & $0.2(0.2-0.7)$ & $<0.001$ & $3.9(0.2-23.2)$ & $0.2(0.2-5.4)$ & 0.002 \\
\hline & \multicolumn{3}{|c|}{$\begin{array}{l}\text { Previuosly untreated patients } \\
(\mathrm{n}=56 ; 25 \text { RRMS and } 31 \text { PrMS) }\end{array}$} & \multicolumn{3}{|c|}{$\begin{array}{l}\text { Previously treated patients } \\
(\mathrm{n}=81 ; 65 \text { RRMS and } 16 \text { PrMS) }\end{array}$} \\
\hline NFL[ng/L] median (IQR) & $849(560-1522)$ & $460(342-680)$ & $<0.001$ & $645(408-1068)$ & $448(275-632)$ & $<0.001$ \\
\hline \multirow[t]{2}{*}{ CXCL13[ng/L] median (IQR) } & $5.9(0.2-30.9)$ & $0.5(0.2-5.8)$ & $<0.001$ & $4.1(0.2-16.3)$ & $0.5(0.2-0.6)$ & $<0.001$ \\
\hline & \multicolumn{3}{|c|}{$\begin{array}{l}\text { Ongoing activity at baseline } \\
\text { (n=63; } 46 \text { RRMS and } 17 \text { PrMS) }\end{array}$} & \multicolumn{3}{|c|}{$\begin{array}{l}\text { No activity at baseline } \\
(\mathrm{n}=74 ; 44 \text { RRMS and } 30 \text { PrMS) }\end{array}$} \\
\hline NFL[ng/L] median (IQR) & $1075(615-2078)$ & $444(298-654)$ & $<0.001$ & $590(381-792)$ & $460(351-650)$ & $<0.001$ \\
\hline \multirow[t]{2}{*}{ CXCL13[ng/L] median (IQR) } & $12.1(2.3-32.5)$ & $0.2(0.2-4.3)$ & $<0.001$ & $0.8(0.2-11.8)$ & $0.2(0.2-1.6)$ & $<0.001$ \\
\hline & \multicolumn{3}{|c|}{$\begin{array}{l}\text { Unchanged or improved EDSS } \\
(\mathrm{n}=119 ; 82 \mathrm{RRMS} \text { and } 37 \text { PrMS) }\end{array}$} & \multicolumn{3}{|c|}{$\begin{array}{l}\text { Worsened EDSS } \\
(\mathrm{n}=18 ; 8 \text { RRMS and } 10 \text { PrMS) }\end{array}$} \\
\hline NFL[ng/L] median (IQR) & $702(409-1374)$ & $447(295-625)$ & $<0.001$ & $720(648-1126)$ & $634(448-838)$ & $<0.001$ \\
\hline CXCL13[ng/L] median (IQR) & $5.4(0.2-21.2)$ & $0.2(0.2-2.9)$ & $<0.001$ & $3.5(0.2-15.5)$ & $0.2(0.2-1.1)$ & 0.010 \\
\hline
\end{tabular}

CXCL13=C-X-C motif chemokine 13, EDSS=Expanded Disability Status Scale, NFL=neurofilament light,

RRMS=relapsing-remitting multiple sclerosis, PrMS=progressive multiple sclerosis, Ongoing activity=relapse

and/or contrast enhancing lesions on magnetic resonance imaging at lumbar puncture

11 NFL and CXCL13 reveal disease activity in clinically and radiologically stable MS 
Table 5. Odds ratio (OR) between increased NFL or CXCL13 levels and NEDA, EDSS worsening, relapse or new MRI lesions

\begin{tabular}{|c|c|c|c|c|c|c|c|}
\hline \multirow[b]{2}{*}{ Prediction of } & \multirow[b]{2}{*}{ Covariates } & \multirow[b]{2}{*}{ B coeficient } & \multirow[b]{2}{*}{ SE } & \multirow[b]{2}{*}{$\mathrm{p}$ value } & \multirow[b]{2}{*}{ OR } & \multicolumn{2}{|c|}{$95 \% \mathrm{CI}$ for $\mathrm{OR}$} \\
\hline & & & & & & Lower & Upper \\
\hline \multirow[t]{2}{*}{ NEDA at follow-up 1} & NFL at baseline & 0,595 & 0,396 & 0,133 & 1,813 & 0,834 & 3,941 \\
\hline & CXCL13 at baseline & $-0,155$ & 0,396 & 0,696 & 0,857 & 0,394 & 1,862 \\
\hline \multirow[t]{2}{*}{ NEDA at follow-up 2} & NFL at baseline & $-0,086$ & 0,417 & 0,837 & 0,918 & 0,405 & 2,078 \\
\hline & CXCL13 at baseline & 0,996 & 0,414 & 0,016 & 2,708 & 1,202 & 6,1 \\
\hline \multirow[t]{2}{*}{ NEDA at follow-up 2} & NFL at follow-up 1 & 0,333 & 0,478 & 0,486 & 1,395 & 0,546 & 3,56 \\
\hline & CXCL13 at follow-up 1 & 1,326 & 0,682 & 0,052 & 3,766 & 0,99 & 14,327 \\
\hline \multirow[t]{2}{*}{ EDSS worsening at follow-up 1} & NFL at baseline & $-0,554$ & 0,548 & 0,312 & 0,575 & 0,197 & 1,681 \\
\hline & CXCL13 at baseline & 0,585 & 0,564 & 0,3 & 1,796 & 0,594 & 5,427 \\
\hline \multirow[t]{2}{*}{ EDSS worsening at follow-up 2} & NFL at baseline & 0,085 & 0,548 & 0,877 & 1,088 & 0,372 & 3,187 \\
\hline & CXCL13 at baseline & $-0,787$ & 0,545 & 0,149 & 0,455 & 0,156 & 1,325 \\
\hline \multirow[t]{2}{*}{ EDSS worsening at follow-up 2} & NFL at follow-up 1 & $-0,025$ & 0,624 & 0,968 & 0,975 & 0,287 & 3,315 \\
\hline & CXCL13 at follow-up 1 & $-0,649$ & 0,78 & 0,405 & 0,522 & 0,113 & 2,411 \\
\hline \multirow[t]{2}{*}{ Relapse at follow-up 1} & NFL at baseline & $-0,991$ & 0,648 & 0,126 & 0,371 & 0,104 & 1,323 \\
\hline & CXCL13 at baseline & 0,906 & 0,637 & 0,155 & 2,474 & 0,71 & 8,616 \\
\hline \multirow[t]{2}{*}{ Relapse at follow-up 2} & NFL at baseline & 1,233 & 0,777 & 0,113 & 3,431 & 0,748 & 15,737 \\
\hline & CXCL13 at baseline & $-0,977$ & 0,744 & 0,189 & 0,376 & 0,088 & 1,618 \\
\hline \multirow[t]{2}{*}{ Relapse at follow-up 2} & NFL at follow-up 1 & 1,003 & 1,131 & 0,375 & 2,727 & 0,297 & 25,047 \\
\hline & CXCL13 at follow-up 1 & $-0,538$ & 1,165 & 0,644 & 0,584 & 0,059 & 5,73 \\
\hline \multirow[t]{2}{*}{ New MR lesions at follow-up 1} & NFL at baseline & $-0,991$ & 0,509 & 0,052 & 0,371 & 0,137 & 1,007 \\
\hline & CXCL13 at baseline & $-0,085$ & 0,471 & 0,856 & 0,918 & 0,365 & 2,313 \\
\hline \multirow[t]{2}{*}{ New MR lesions at follow-up 2} & NFL at baseline & $-1,172$ & 0,576 & 0,042 & 0,31 & 0,1 & 0,959 \\
\hline & CXCL13 at baseline & $-0,372$ & 0,507 & 0,463 & 0,689 & 0,255 & 1,863 \\
\hline \multirow[t]{2}{*}{ New MR lesions at follow-up 2} & NFL at follow-up 1 & $-1,335$ & 0,531 & 0,012 & 0,263 & 0,093 & 0,745 \\
\hline & CXCL13 at follow-up 1 & $-0,805$ & 0,717 & 0,262 & 0,447 & 0,11 & 1,824 \\
\hline
\end{tabular}

CXCL13=C-X-C motif chemokine 13, EDSS=Expanded Disability Status Scale, MRI=magnetic resonance imaging, NEDA=No evidence of disease activity, NFL=neurofilament light

NEDA was defined as no relapse, no increase in disability measured by EDSS, no new or enlarging T2 lesions and no Gd contrast enhancing lesions on MRI. EDSS worsening was present if EDSS increased $\geq 1.0$-point if baseline EDSS was 0.0-5.0 or $\geq 0.5$ if baseline EDSS was $\geq 5.5$, and was considered unchanged if EDSS changed less. New MRI lesions were present if there were contrast or new or enlarging non-contrast enhancing lesions 
Figure 1. Study design

\begin{tabular}{|l|l|l|l|}
\hline $\begin{array}{l}\text { Baseline } \\
\text { LP } \\
\begin{array}{l}\text { EDSS } \\
\text { Ongoing disease activity } \\
\text { (Gd+ lesions or relapse) }\end{array}\end{array}$ & $\begin{array}{l}\text { Follow-up } \\
\text { Brain MRI } \\
\text { LP } \\
\text { EDSS } \\
\text { Ongoing disease activity (Gd+ } \\
\text { lesions or relapse) } \\
\text { NEDA (Gd+ lesions or } \\
\text { new/enlarging T2 lesions or } \\
\text { relapse or EDSS worsening) }\end{array}$ & $\begin{array}{l}\text { Follow-up } \\
\text { Brain MRI } \\
\text { EDSS } \\
\text { NEDA (Gd+ lesions or } \\
\text { new/enlarging T2 lesions or } \\
\text { relapse or EDSS worsening) }\end{array}$ \\
\hline
\end{tabular}


Figure 2. Levels of CXCL 13 and NFL

A
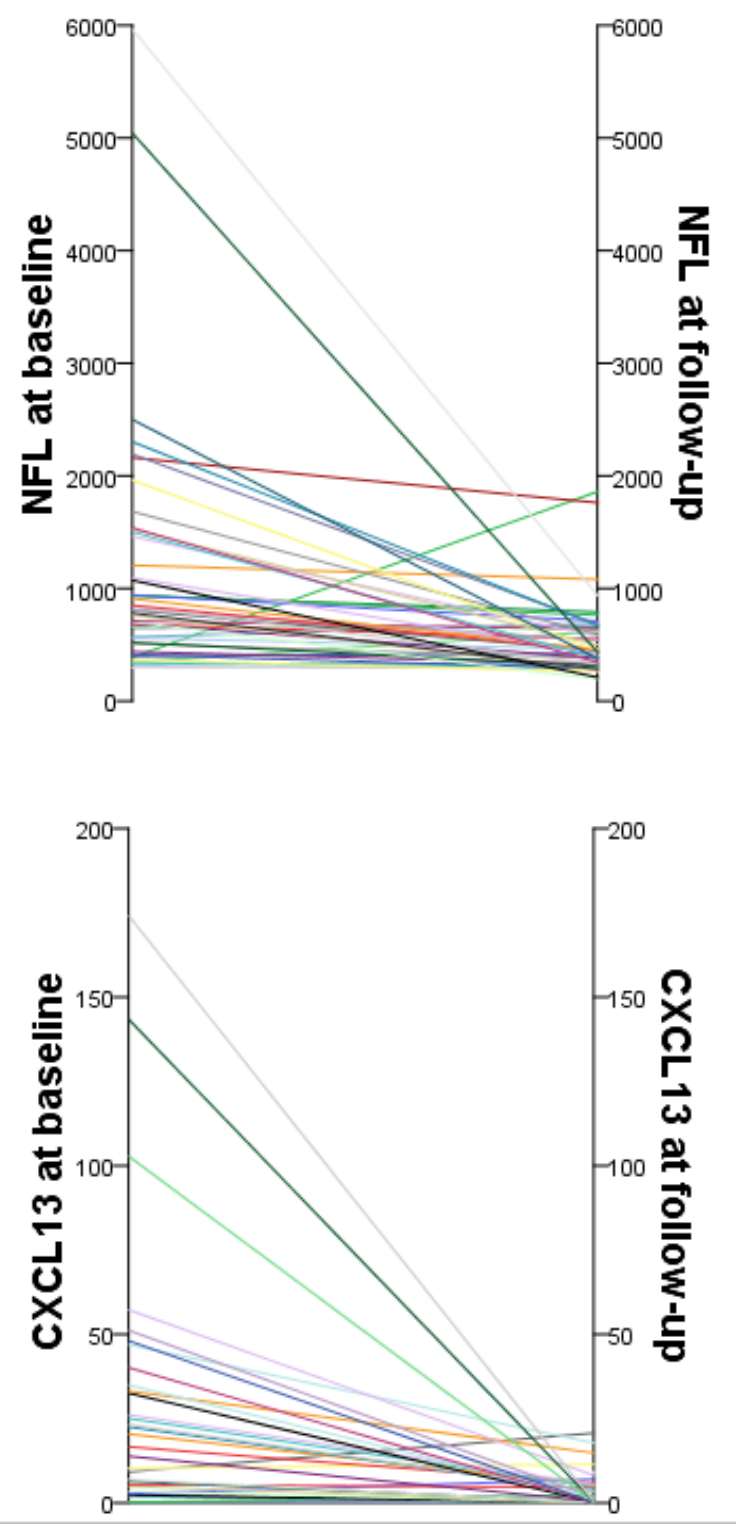

B
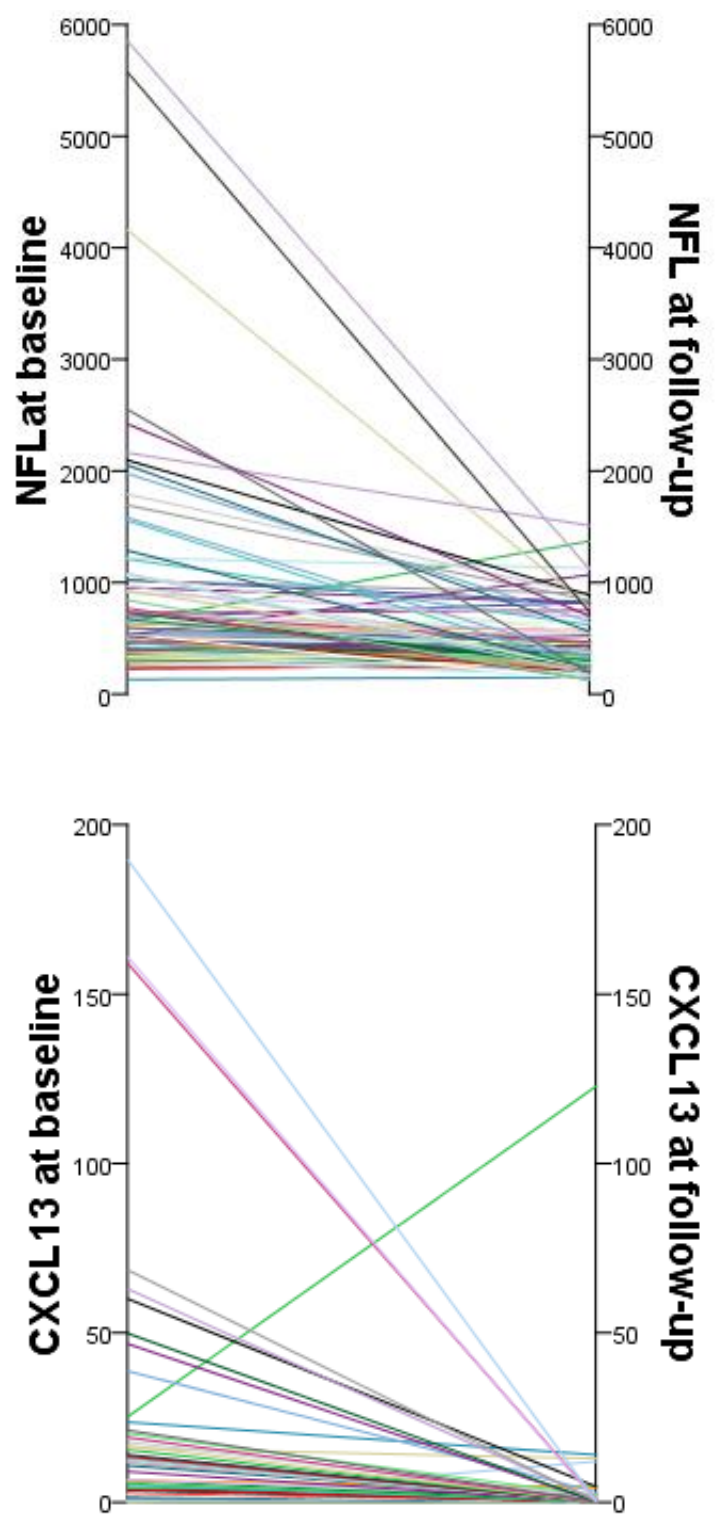


\section{Figure legend}

\section{Figure 1. Study design}

Baseline, first follow-up after median 12 months, and second follow-up after median 27 months from baseline with clinical and MRI measures examinations and associated evaluation of patients.

EDSS=Expanded Disability Status Scale, LP=lumbar puncture, MRI=magnetic resonance imaging, NEDA=no evidence of disease activity.

\section{Figure 2. Levels of CXCL 13 and NFL}

The levels of CXCL13 (ng/L) and NFL (ng/L) in CSF are shown in previously untreated patients (A) and in previously treated patients (B) with a median time of 12 months of follow up between these two samplings.

(A) Previously untreated patients $(n=48)$, RRMS ( $n=23)$ and PrMS $(n=25)$. NFL levels decreased from 810 (IQR 520-1522) to 452 (IQR 337-660) ng/L and CXCL!3 levels decreased from 5.9 (IQR 0.2-25.8) to 0.2 (IQR 0.2-4.4) ng/L ( $\mathrm{p}<0.001$ and $\mathrm{p}<0.001$, respectively). Two patients with NFL >6000 ng/L were excluded in this figure (in both NFL levels were decreased).

(B) Previously treated patients $(n=79)$, RRMS ( $n=65)$ and PrMS $(n=14)$. NFL levels decreased from 645 (IQR 407-1073) to 443 (IQR 266-631) ng/L and CXCL!3 levels decreased from 4.1 (IQR 0.2-15.9) to 0.2 (IQR 0.2-0.5) ng/L ( $<<0.001$ and $\mathrm{p}<0.001$, respectively). One patients with NFL >6000 ng/L was excluded in this figure (NFL levels were decreased). One patients with CXCL13 > $200 \mathrm{ng} / \mathrm{L}$ was excluded in this figure (CXCL13 levels were decreased).

\section{Acknowledgements}

The study was supported by grants from the Swedish State Support for Clinical Research (ALFGBG), NEURO Sweden, NEURO Gothenburg, Edith Jacobsons Foundation and Helena Ahlin's Foundation. HZ is a Wallenberg Scholar supported by grants from the Swedish Research Council (\#2018-02532), the European Research Council (\#681712), Swedish State Support for Clinical Research (\#ALFGBG-720931), the Alzheimer Drug Discovery Foundation (ADDF), USA (\#201809-2016862), and the UK Dementia Research Institute at UCL. 


\section{References:}

Barro, C., Benkert, P., Disanto, G. et al. (2018) Serum neurofilament as a predictor of disease worsening and brain and spinal cord atrophy in multiple sclerosis. Brain, 141, 23822391.

Bergman, J., Dring, A., Zetterberg, H., Blennow, K., Norgren, N., Gilthorpe, J., Bergenheim, T. and Svenningsson, A. (2016) Neurofilament light in CSF and serum is a sensitive marker for axonal white matter injury in MS. Neurol Neuroimmunol Neuroinflamm, 3, e271.

Bjerke, M., Jonsson, M., Nordlund, A. et al. (2014) Cerebrovascular Biomarker Profile Is Related to White Matter Disease and Ventricular Dilation in a LADIS Substudy. Dement Geriatr Cogn Dis Extra, 4, 385-394.

Cotton, F., Weiner, H. L., Jolesz, F. A. and Guttmann, C. R. (2003) MRI contrast uptake in new lesions in relapsing-remitting MS followed at weekly intervals. Neurology, 60 , 640-646.

Disanto, G., Barro, C., Benkert, P. et al. (2017) Serum Neurofilament light: A biomarker of neuronal damage in multiple sclerosis. Ann Neurol, 81, 857-870.

Dubuisson, N., Puentes, F., Giovannoni, G. and Gnanapavan, S. (2017) Science is $1 \%$ inspiration and 99\% biomarkers. Mult Scler, 1352458517709362.

Giovannoni, G., Turner, B., Gnanapavan, S., Offiah, C., Schmierer, K. and Marta, M. (2015) Is it time to target no evident disease activity (NEDA) in multiple sclerosis? Mult Scler Relat Disord, 4, 329-333.

Gunnarsson, M., Malmestrom, C., Axelsson, M. et al. (2011) Axonal damage in relapsing multiple sclerosis is markedly reduced by natalizumab. Ann Neurol, 69, 83-89.

Hakansson, I., Tisell, A., Cassel, P., Blennow, K., Zetterberg, H., Lundberg, P., Dahle, C., Vrethem, M. and Ernerudh, J. (2018) Neurofilament levels, disease activity and brain volume during follow-up in multiple sclerosis. J Neuroinflammation, 15, 209.

Kazanietz, M. G., Durando, M. and Cooke, M. (2019) CXCL13 and Its Receptor CXCR5 in Cancer: Inflammation, Immune Response, and Beyond. Front Endocrinol (Lausanne), 10, 471.

Khademi, M., Kockum, I., Andersson, M. L., Iacobaeus, E., Brundin, L., Sellebjerg, F., Hillert, J., Piehl, F. and Olsson, T. (2011) Cerebrospinal fluid CXCL13 in multiple sclerosis: a suggestive prognostic marker for the disease course. Mult Scler, 17, 335343.

Kowarik, M. C., Cepok, S., Sellner, J., Grummel, V., Weber, M. S., Korn, T., Berthele, A. and Hemmer, B. (2012) CXCL13 is the major determinant for B cell recruitment to the CSF during neuroinflammation. J Neuroinflammation, 9, 93.

Kuhle, J., Barro, C., Andreasson, U. et al. (2016) Comparison of three analytical platforms for quantification of the neurofilament light chain in blood samples: ELISA, electrochemiluminescence immunoassay and Simoa. Clin Chem Lab Med.

Kuhle, J., Kropshofer, H., Haering, D. A. et al. (2019) Blood neurofilament light chain as a biomarker of MS disease activity and treatment response. Neurology, 92, e1007e1015.

Kurtzke, J. F. (1983) Rating neurologic impairment in multiple sclerosis: an expanded disability status scale (EDSS). Neurology, 33, 1444-1452.

Lycke, J. and Zetterberg, H. (2017) The role of blood and CSF biomarkers in the evaluation of new treatments against multiple sclerosis. Expert Rev Clin Immunol, 13, 1143-1153. 
Malmestrom, C., Haghighi, S., Rosengren, L., Andersen, O. and Lycke, J. (2003)

Neurofilament light protein and glial fibrillary acidic protein as biological markers in MS. Neurology, 61, 1720-1725.

Meyer-Moock, S., Feng, Y. S., Maeurer, M., Dippel, F. W. and Kohlmann, T. (2014) Systematic literature review and validity evaluation of the Expanded Disability Status Scale (EDSS) and the Multiple Sclerosis Functional Composite (MSFC) in patients with multiple sclerosis. BMC Neurol, 14, 58.

Mitsdoerffer, M. and Peters, A. (2016) Tertiary Lymphoid Organs in Central Nervous System Autoimmunity. Front Immunol, 7, 451.

Norgren, N., Rosengren, L. and Stigbrand, T. (2003) Elevated neurofilament levels in neurological diseases. Brain Res, 987, 25-31.

Novakova, L., Axelsson, M., Khademi, M., Zetterberg, H., Blennow, K., Malmestrom, C., Piehl, F., Olsson, T. and Lycke, J. (2017a) Cerebrospinal fluid biomarkers as a measure of disease activity and treatment efficacy in relapsing-remitting multiple sclerosis. J Neurochem, 141, 296-304.

Novakova, L., Zetterberg, H., Sundstrom, P. et al. (2017b) Monitoring disease activity in multiple sclerosis using serum neurofilament light protein. Neurology, 89, 2230-2237.

Pilz, G., Sakic, I., Wipfler, P., Kraus, J., Haschke-Becher, E., Hitzl, W., Trinka, E. and Harrer, A. (2020) Chemokine CXCL13 in serum, CSF and blood-CSF barrier function: evidence of compartment restriction. Fluids Barriers CNS, 17, 7.

Sejbaek, T., Nielsen, H. H., Penner, N., Plavina, T., Mendoza, J. P., Martin, N. A., Elkjaer, M. L., Ravnborg, M. H. and Illes, Z. (2019) Dimethyl fumarate decreases neurofilament light chain in CSF and blood of treatment naive relapsing MS patients. J Neurol Neurosurg Psychiatry, 90, 1324-1330.

Sellebjerg, F., Bornsen, L., Khademi, M., Krakauer, M., Olsson, T., Frederiksen, J. L. and Sorensen, P. S. (2009) Increased cerebrospinal fluid concentrations of the chemokine CXCL13 in active MS. Neurology, 73, 2003-2010.

Teunissen, C. E. and Khalil, M. (2012) Neurofilaments as biomarkers in multiple sclerosis. Mult Scler, 18, 552-556.

Teunissen, C. E., Petzold, A., Bennett, J. L. et al. (2009) A consensus protocol for the standardization of cerebrospinal fluid collection and biobanking. Neurology, 73, 19141922.

Thebault, S., D, R. T., Lee, H., Bowman, M., Bar-Or, A., Arnold, D. L., H, L. A., TabardCossa, V. and Freedman, M. S. (2019) High serum neurofilament light chain normalizes after hematopoietic stem cell transplantation for MS. Neurol Neuroimmunol Neuroinflamm, 6, e598.

Trapp, B. D., Peterson, J., Ransohoff, R. M., Rudick, R., Mork, S. and Bo, L. (1998) Axonal transection in the lesions of multiple sclerosis. The New England journal of medicine, 338, 278-285.

Ziemssen, T., De Stefano, N., Sormani, M. P., Van Wijmeersch, B., Wiendl, H. and Kieseier, B. C. (2015) Optimizing therapy early in multiple sclerosis: An evidence-based view. Mult Scler Relat Disord, 4, 460-469. 